\title{
Analgesic prescriptions received by patients before commencing the BOA model of care for osteoarthritis: a Swedish national registry study with matched reference and clinical guideline benchmarking
}

\author{
Allan ABBOTT ${ }^{1}$, Kristin GUSTAFSSON ${ }^{1,2}$, Caddie ZHOU ${ }^{3}$, Ola ROLFSON ${ }^{4}$, and Gunilla Limbäck SVENSSON ${ }^{4,5}$ \\ 1 Unit of Physiotherapy, Department of Health, Medicine and Caring Sciences, Linköping University, Linköping; ${ }^{2}$ Department of Physiotherapy, \\ Rehabilitation Centre, Ryhov County Hospital, Jönköping; ${ }^{3}$ Centre of Registries Västra Götaland, Gothenburg; ${ }^{4}$ Department of Orthopaedics, Sahlgrenska \\ Academy, Institute of Clinical Sciences, University of Gothenburg, Gothenburg; ${ }^{5}$ BOA Registry, Centre of Registries, Västra Götaland, Gothenburg, \\ Sweden \\ Correspondence: allan.abbott@liu.se \\ Submitted 2021-04-28. Accepted 2021-09-29.
}

Background and purpose - Swedish clinical guidelines for osteoarthritis (OA) prioritize patient education, exercise, and-if necessary - weight reduction before considering adjunct pharmacological intervention. Contrariwise, we investigated the proportion and type of dispensed analgesic prescriptions in Sweden received by patients during 3 years before commencing non-pharmacological primary care interventions for OA (2008-2016) compared with the general population. Furthermore, we analyzed the proportion of analgesic prescriptions dispensed before (2008-2012) compared with after (2012-2016) guideline publication in terms of concordance with clinical guideline recommendations.

Patients and methods - Patients with hip or knee OA $(\mathrm{n}=72,069)$ from the Better Management of OA national quality register receiving non-pharmacological interventions in primary care between 2008 and 2016 were included (OA cohort). An age, sex, and residence matched reference cohort $(n=216,207)$ was formed from the Swedish Total Population Register. Based on a period 3 years prior to inclusion in the OA cohort, Swedish Prescribed Drug Register data was linked to both the OA and reference cohorts.

Results - Compared with the reference cohort, a distinctly larger proportion of the OA cohort had dispensed prescriptions for most types of analgesics, increasing exponentially each year prior to commencing non-pharmacological intervention. Since guideline publication, the proportion of the OA cohort having no dispensed prescription analgesics prior to non-pharmacological primary care intervention concordantly increased by $5.0 \%$ (95\% CI 4.2-5.9). Furthermore, dispensed prescriptions concordantly decreased for non-selective NSAIDs $-8.6 \%$ (CI -9.6 to -7.6 ), weak opioids $-6.8 \%$ (CI -7.7 to -5.9 ), glucosamine $-9.5 \%$ (CI -9.8 to -8.8 ). and hyaluronic acid $-1.6 \%$ (CI -1.8 to -1.5$)$ but discordantly increased for strong opioids $2.8 \%$ (CI 2.1-3.4) and glucocorticoid intra-articular injection for hip OA $2.1 \%$ (CI 1.0-3.1).

Interpretation - In Sweden, dispensed prescription of analgesics commonly occurred before initiating non-pharmacological primary care interventions for OA but reduced modestly after guideline publication, which prioritizes nonpharmacological before pharmacological interventions. Additional modest improvements occurred in the steppedcare prioritization of analgesic prescription types. However, future strategies are required to curb an increase of strong opioids prescription for OA and glucocorticoid intra-articular injection for hip OA.

Osteoarthritis $(\mathrm{OA})$ is among the highest ranked contributors to disability globally (1). In Sweden, the Better Management of patients with OA (BOA) model of care is intended as a first step in primary care providing patient education, exercise, and-if necessary - weight reduction interventions in a supported self-management program. Since 2008, the BOA model of care has been successively implemented in Swedish primary care nationally (2). By the end of 2016, the BOA national quality register had a national coverage of $84 \%$ of clinics offering the BOA model of care, with a data registration completeness of $76 \%$ (3).

Swedish clinical guidelines for hip and knee OA were first published in 2012 (4). These guidelines have a stepped care structure where in a first step in primary care, non-pharmacological interventions such as patient education, exercise, 
and - if necessary - weight reduction were prioritized. If the effect of first-step non-pharmacological primary care interventions is not sufficient, the 2012 guidelines recommended adjunct interventions such as walking aids and stepped care trialing of analgesic medications as a second step (4). Nonsteroidal anti-inflammatory drugs (NSAIDs) or acetaminophen (paracetamol) were recommended as appropriate analgesics. In the case of insufficient effect, short-term trial of weak opioids and lastly strong opioids were considered as final alternatives. The Swedish guidelines also recommended against the prescription of glucosamine and hyaluronic acid injection for hip or knee OA and glucocorticoid injection for hip OA.

Contrariwise, no previous studies have investigated the prevalence of pharmacological interventions occurring before non-pharmacological intervention in Swedish routine primary care for OA. We investigated proportion and type of dispensed analgesic prescriptions in Sweden received by patients during the 3 years before commencing non-pharmacological primary care interventions for OA (2008-2016) compared with the general population. Furthermore, we analyzed the proportion of dispensed analgesic prescriptions before (2008-2012) compared with after (2012-2016) guideline publication in terms of concordance with clinical guideline recommendations.

\section{Patients and methods}

\section{Study design}

This is a national registry linkage study of cross-sectional design with data from the BOA national quality register (2), the Swedish Total Population Register (TPR) (5), the Swedish Prescribed Drug Register (SPDR) (6), and descriptive data from the Longitudinal Integration Database for Health Insurance and Labour Market Studies (LISA) (7). This study is reported in line with the RECORD-PE checklist.

\section{Description of data sources and linkage}

\section{OA cohort}

All 75,482 patient registrations in the BOA national quality register between January 1, 2008 and December 31, 2016 for baseline data collected upon commencement of the BOA model of care were initially included in the OA cohort. Through use of the personal identity number (PIN) assigned to all Swedish residents, linkage between the OA cohort and TPR could be performed by the government agency Statistics Sweden. This resulted in the OA cohort being reduced to 75,415 due to 14 patients in the OA cohort not having data in the TPR and 53 patients' personal identification numbers occurring more than once in the OA cohort. Furthermore, the cohort was further reduced to 72,069 after excluding 3,346 patients for whom other joints than the hip or the knee were registered as the worst affected by OA (Figure). The final cohort of patients have confirmed clinical and/or radiographic diagnostic criteria for hip or knee OA in line with the national guidelines

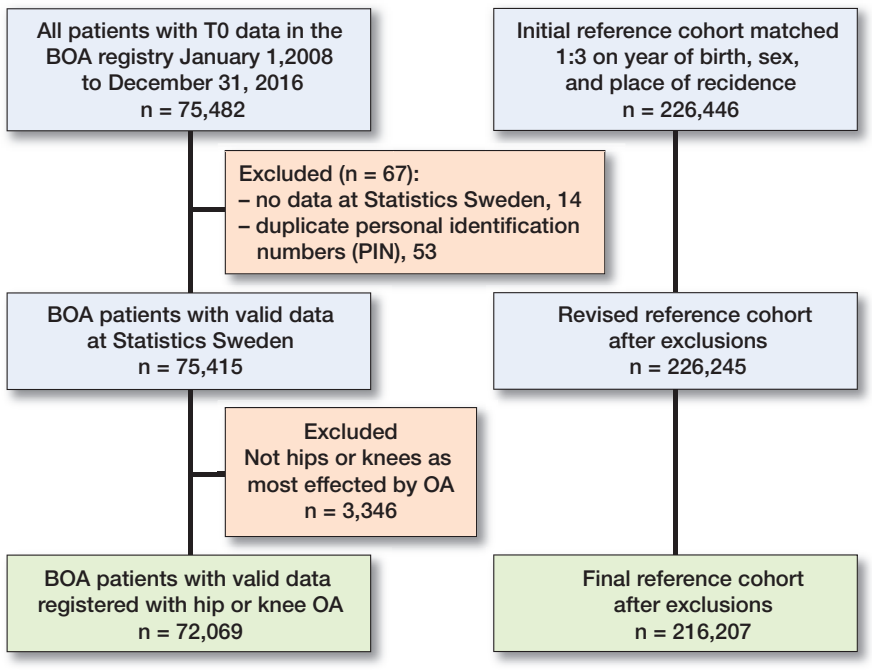

Flow chart displaying the included OA cohort and matched reference cohort. Adapted with permission from Gustafsson et al. (11)

(4) and Altman et al. $(\mathbf{8 , 9 )}$ as well as exclusion of differential diagnoses such as tumor, fracture, inflammatory joint disease, and chronic widespread pain. Furthermore, patients who had received total joint replacement within the previous 12 months or other surgery of the knee or hip joint within the previous 3 months and inability to read or understand Swedish were also excluded from the OA cohort. Data on the worst affected joint for OA, numeric rating scale for pain (10), and Charnley score were extracted from the BOA national quality register for the OA cohort.

\section{Reference cohort}

The government agency, Statistics Sweden, randomly selected a reference cohort $(n=226,446)$ from Swedish residents in the TPR through 3:1 matching with the patient registrations in the BOA national quality register based on year of birth, sex, and regional area of residence in Sweden (Figure). The matching ensured that the person with the same birth year in the reference population is alive at the time of the index person's inclusion in the OA cohort and that all participants were living in Sweden for the entire 3 years of the study time window. After exclusion criteria were applied producing the final OA cohort, the final matched reference cohort consisted of 216,207 individuals. The reference cohort had never been included in the BOA national quality register. The TPR is often used to form general population reference cohorts because it has almost $100 \%$ national coverage and $100 \%$ data registration completeness (5).

Details concerning the selection of the OA cohort, and the creation and matching of the reference cohort from the TPR have previously been described by Gustafsson et al. (11) (Figure). For the OA and reference cohorts, Statistics Sweden extracted data on age, sex, marital status (married, including registered partner or not married), and whether the indi- 
vidual was born outside Sweden (yes or no) from the TPR. Furthermore, data was extracted for educational level (low = $\leq 9$ years, medium $=10-14$ years, and high $=\geq 15$ years) and disposable income from LISA.

Data was anchored to the date in time for baseline in the BOA national quality register (T0). Statistics Sweden extracted individual-level data from the SPDR for the OA cohort and their matched reference cohort regarding a period 3 years before baseline in the BOA national quality register. Data for prescription medications that have been dispensed was extracted from the SPDR. Prescription-free medicines or medicines that are given during hospital care are, however, not included in the SPDR. Furthermore, the indication for the prescription (e.g., if analgesic medications are prescribed for osteoarthritis, or for other painful conditions) is not included in the SPDR. Medications are registered in the SPDR with their Anatomic Therapeutic Chemical classification system (ATC) code. ATC is a classification system wherein drugs are divided into groups, with several subgroups, based on their indication area (12). For this study we included medications covering ATC codes for (1) NSAIDs (non-selective per os, selective COX inhibitors per os, non-selective topical), (2) paracetamol, (3) opioids (weak, strong), (4) other joint-related drugs with intra-articular administration, and (5) antiepileptic drug for neuropathic pain as described in Table 1 (see Supplementary data).

\section{Statistics}

Descriptive statistics were used to describe the cohort's demographics, socioeconomic factors, and dispensed prescriptions for analgesics. Means and standard deviations (SD) or median and interquartile range (IQR) were used for continuous data, while frequencies and proportions were used for categorical data. The proportion of individuals with dispensed prescriptions for analgesics per year in the OA cohort 3 years prior to commencing the BOA model of care for symptomatic hip or knee OA was compared with the matched reference cohort using $95 \%$ confidence intervals (CI) and Wald test from logistical regression adjusted for the matching variables age, sex, and regional area. Furthermore, the same method was used to compare the proportion of dispensed analgesics prescriptions in the OA and matched reference cohorts before and after the publication of clinical guideline recommendations for $\mathrm{OA}$ in 2012. A p-value $\leq 0.05$ is considered statistically significant. All statistical analyses were performed with SAS 9.4 TS Level 1MS and IBM SPSS Statistics for Windows, Version 25.0 (IBM Corp, Armonk, NY, USA).

\section{Ethics, funding, and potential conflicts of interest}

Ethical approval for this study, in compliance with the Helsinki Declaration, has been granted by the Regional Ethical Review Board in Gothenburg, Sweden (14-03-2017, dnr 1059-16). Ethical approval allowed data collected and housed by Swedish national registries to be accessed on a secure online server
Table 2. OA cohort and reference cohort characteristics. Values are count $(\%)$ unless otherwise specified

\begin{tabular}{|c|c|c|}
\hline & $\begin{array}{l}\text { OA cohort } \\
n=72,069\end{array}$ & $\begin{array}{c}\text { Reference cohort } \\
n=216,207\end{array}$ \\
\hline Age, mean (SD) & $66.4(9.6)$ & $66.4(9.6)$ \\
\hline Women & $49,494(69)$ & $148,482(69)$ \\
\hline \multicolumn{3}{|l|}{ Worst affect joint in OA cohort } \\
\hline Knee & $49,366(68)$ & \\
\hline Hip & $22,703(32)$ & \\
\hline Pain NRS, mean (SD) & $5.4(2.0)$ & \\
\hline Missing & $621(<0.1)$ & \\
\hline \multicolumn{3}{|l|}{ Charnley score } \\
\hline A & $27,242(38)$ & \\
\hline B & $13,471(18)$ & \\
\hline C & $30,918(43)$ & \\
\hline Missing & $438(<0.1)$ & \\
\hline Born outside Sweden & $6,474(9)$ & $28,554(13)$ \\
\hline Missing & 0 & $12(<0.1)$ \\
\hline Married & $42,359(59)$ & $117,616(54)$ \\
\hline Missing & $9(<0.1)$ & $162(0.1)$ \\
\hline \multicolumn{3}{|l|}{ Educational level a } \\
\hline Low ( $\leq 9$ years) & $16,276(23)$ & $61,212(28)$ \\
\hline Medium (10-14 years) & $43,492(60)$ & $119,416(55)$ \\
\hline High ( $\geq 15$ years) & $12,111(17)$ & $33,260(15)$ \\
\hline Missing & $190(0.3)$ & $2,319(1.1)$ \\
\hline \multicolumn{3}{|l|}{ Disposable annual income (€) } \\
\hline year of T0, median (IQR) $\mathbf{b}^{\prime}$ & $20,022(11,936)$ & $18,559(12,516)$ \\
\hline Missing & $4(<0.1)$ & $132(0.1)$ \\
\hline
\end{tabular}

$\mathrm{TO}=$ Baseline in the BOA national quality register

a For the participants with TO during 2016, the highest achieved level up to 2015 was used.

b Data only reported for those in the cohorts with TO between 2008-2015. During 2008-2015, the average exchange rate was $1 €=9.3$ SEK

for linkage and analysis by project investigators but data sharing outside of the secure online server was not possible. The study was financially supported by AFA Insurance, Sweden (160176). The authors have no conflicts of interest.

\section{Results}

Demographics and socioeconomic characteristics for the study cohorts showed no statistically significant differences (Table 2). The OA cohort had a mean pain NRS of 5.4 (SD 2.0) while $38 \%$ had a Charnley score A, $19 \%$ a Charnley score $\mathrm{B}$, and $43 \%$ a Charnley score C.

In the OA cohort $0-3$ years prior to commencing the BOA model of care, NSAIDs were the most dispensed analgesic prescription (non-selective per os 25\%) (Table 3). Dispensed analgesic prescriptions were statistically significantly higher than the reference cohort for all forms of NSAIDs, paracetamol, weak opioids, and other joint-pain-related analgesics. Between 3 and 2 years prior to commencing the BOA model of care, the OA cohort's dispensed prescriptions for the same analgesics remained statistically significantly higher and increased by a factor of 1.2 compared with the reference cohort. 
Table 3. Comparisons of proportion and type of dispensed analgesic prescriptions in Sweden received by patients before commencing non-pharmacological primary care interventions for osteoarthritis $(O A, n=72,069)$ compared a general population cohort $($ Ref. $n=216,207)$

\begin{tabular}{|c|c|c|c|c|c|c|c|c|c|c|c|}
\hline \multirow[b]{3}{*}{ Analgesic medications } & \multicolumn{10}{|c|}{$\begin{array}{l}\text { Proportion of individuals with dispensed prescriptions for analgesics prior to } \\
\text { commencing the BOA model of care for symptomatic hip or knee OA }\end{array}$} & \multirow[b]{3}{*}{$\mathrm{C} / \mathrm{B}$} \\
\hline & \multicolumn{3}{|c|}{ Between 3 and 2 years } & \multicolumn{3}{|c|}{ Between 2 and 1 years } & \multicolumn{4}{|c|}{ Between 1 and 0 year } & \\
\hline & $\mathrm{OA}$ & Ref. & A. $\Delta(95 \% \mathrm{Cl})$ & OA & Ref. & B. $\Delta(95 \% \mathrm{Cl})$ & $\mathrm{B} / \mathrm{A}$ & $\mathrm{OA}$ & Ref. & C. $\Delta(95 \% \mathrm{Cl})$ & \\
\hline NSAIDs non-selective per os & 25 & 16 & & 26 & 15 & & 1 & 41 & 15 & & 2.5 \\
\hline $\begin{array}{l}\text { NSAIDs non-selective topical } \\
\text { NSAIDs selective COX }\end{array}$ & 1.8 & 1.3 & $0.5(0.4$ to 0.6$)$ & 1.9 & 1.3 & & 1.2 & 2.8 & 1.3 & & 2.5 \\
\hline inhibito & 2.9 & 1.6 & $1.3(1.2$ to 1.4$)$ & 3.2 & 1.6 & $1.6(1.5$ to 1.7$)$ & 1.2 & 5.8 & 1.7 & 4.1 (3.9 to 4.3$)$ & 2.6 \\
\hline Parace & 23 & 18 & & 26 & 20 & & 1. & 41 & 21 & & 3.2 \\
\hline Weak & 11 & 9.7 & 1.7 (1.4 to 2.0$)$ & 11 & 9.3 & $2.0(1.7$ to 2.3$)$ & 1.2 & 16 & 9.0 & 6 to 7.2$)$ & 3.5 \\
\hline Strons & 4.1 & 4.2 & $-0.1(-0.3$ to 0.1$)$ & 4.6 & 4.8 & $-0.2(-0.4$ to 0.0$)$ & -2.0 & 6.7 & 5.8 & .7 to 1.1$)$ & 4.5 \\
\hline Antiepileptics & 1.3 & 1.4 & $-0.1(-0.2$ to 0.0$)$ & 1.5 & 1.6 & $-0.1(-0.2$ to 0.0$)$ & 1.0 & 1.7 & 1.8 & $-0.1(-0.2$ to 0.0$)$ & 1.0 \\
\hline Other joint pain related & & & & & & & & & & & \\
\hline analgesics & 8.6 & 5.9 & 2.7 (2.5 to 2.9 ) & 8.7 & 5.9 & 2.8 (2.6 to 3.0 ) & 1.0 & 10 & 6.1 & 4.3 (4.1 to 4.5$)$ & 1.5 \\
\hline
\end{tabular}

$\Delta$ Difference between $\mathrm{OA}$ and Ref.

For the year prior to commencing the BOA model of care, the OA cohort's dispensed prescriptions for the same analgesics remained statistically significantly higher and increased most notably by a factor of 1.5-3.5 times compared with the reference cohort. In addition, dispensed prescriptions for strong opioids in the OA cohort became statistically significantly but modestly higher compared with the reference cohort $(0.9 \%$, CI 0.7-1.1), increasing by a factor of 4.5 times. There were negligible differences between the OA and reference cohorts regarding dispensed prescriptions of antiepileptics.

The proportion of individuals with no dispensed analgesic prescriptions prior to commencing the BOA model of care statistically significantly increased by $5.0 \%$ (CI 4.2-5.9) from $18 \%$ to $23 \%$ after the guidelines were released (Table 4). Furthermore, the proportion of individuals with dispensed prescriptions statistically significantly decreased: $-8.6 \%$ (CI -9.6 to -7.6$)$ for NSAIDs non-selective per os, $-1.0 \%$ (CI -1.5 to -0.6 ) for NSAIDs non-selective topical, $-0.2 \%$ (CI -0.8 to 0.4 ) for NSAIDs selective COX inhibitors per os, $-6.8 \%$ (CI -7.7 to -5.9 ) for weak opioids, $-9.5 \%$ (CI -9.8 to -8.8 ) for glucosamine, and $-1.6 \%$ (CI -1.8 to -1.5 ) for hyaluronic acid injection intra-articular injection. Conversely, the proportion of dispensed prescriptions increased statistically significantly for strong opioids $2.7 \%$ (CI 2.1-3.4) and for glucocorticoid injection for hip OA 2.1\% (CI 1.0-3.1). Proportions of dispensed prescriptions were unchanged for paracetamol, NSAIDs selective COX inhibitors per os and intra-articular glucocorticoid injection for knee OA.

\section{Discussion}

In the OA cohort, there was a larger proportion of dispensed prescriptions for most analgesics, increasing exponentially each year from 3 years prior to commencing the BOA model of care compared with the reference cohort. This was most nota- ble for NSAIDs and paracetamol over time but even evident for opioid prescription in the later phase before commencement of the BOA model of care. Previous literature based on the Osteoarthritis Initiative database $(n=987)$ has also reported change in analgesic type over time but no change in the proportion of patients using analgesics over time (13). In our study, antiepileptic analgesic medications such as gabapentinoids had negligible differences in the proportion dispensed in the OA cohort compared with the reference cohort. In contrast, a United Kingdom national cohort study in primary care reported increased gabapentinoid prescribing for patients between 1995 and 2015. Diagnostic codes could be partly matched to prescription data, indicating that prescriptions were possibly attributable to treating OA-related joint pain but to a larger extent for a broad array of neuropathic pain conditions (14).

In discordance with the stepped care structure of the Swedish national guidelines for OA, as a first step only $18 \%$ of patients with OA commenced exercise, education, and weight management before analgesics were prescribed. In other words, over $80 \%$ in the OA cohort had already commenced pharmacological interventions with approximately 30 percentage points higher dispensed analgesic prescriptions than the reference cohort. During the period after publication of the guidelines 2012-2016, the proportion of patients receiving first-step non-pharmacological interventions before the consideration of analgesic prescription in the OA cohort had increased by 5.1 percentage points compared with the period 2008-2012. Further strategies are therefore required in Sweden to improve healthcare practitioner compliance with the guidelines by providing analgesic prescriptions as an adjunct only if first-step interventions do not give satisfactory results alone.

Exercise has been shown to have a similar analgesic effect but better cost-effectiveness than prescription analgesic medications for pain secondary to knee OA $(\mathbf{1 5 , 1 6 )}$. The all-cause mortality reducing effects of exercise (17) and the potentially harmful side effects of prolonged analgesic use (18-20) 
Table 4. Comparisons of the proportion of dispensed analgesic prescriptions in terms of clinical guideline recommendations before (01/01/2008-31/05/2012) compared to after (01/06/2012-31/12/2016) guideline publication

Guideline recommendations for hip or knee

OA according to stepped care priority

- Dispensed analgesic prescriptions
OA cohort $(n=72,069)$

Before After

$\begin{array}{lcc}\text { Total no. }= & 10,862 & 61,207 \\ \text { Knees }= & 7,539 & 41,827\end{array}$

Hips =
Reference cohort $(n=216,207)$

Before After

$32,586 \quad 183,621$

$2,617 \quad 125,481$

$9,969 \quad 58,140 \quad \Delta(95 \% \mathrm{Cl})$

1. A moderate-strong priority recommendation for first step interventions such as exercise $(3 / 10)^{a}$, patient education $(6 / 10)^{a}$, and weight management $(5 / 10)^{a}$, before considering adjunct pharmacological interventions

- No dispensed analgesic

2. A low-moderate priority recommendation $\left(6-7 / 10^{a}\right.$ for NSAIDs (selective COX inhibitors per os) as an adjunct treatment if first step interventions do not give satisfactory results - COX-2 inhibitors

3. A low-moderate priority $(7 / 10)^{a}$ recommendation for NSAIDs (nonselective per os) as an adjunct treatment if first step interventions have not given satisfactory results - NSAIDs

4. A low priority recommendation $(7 / 10)^{a}$ for intra-articular glucocorticoid injection for knee OA as an adjunct treatment if first step interventions do not give satisfactory results

- Intra-articular glucocorticoid injection for knee OA

5. A low priority recommendation $(8 / 10)^{a}$ for paracetamol as an adjunct treatment if first step interventions do not give satisfactory results

- Paracetamol

6. A low priority recommendation $(9 / 10)^{a}$ for weak opioids as an adjunct treatment if first step interventions do not give satisfactory results

- Weak opioids

7. Weakest priority recommendation (10/10)\# for NSAIDs (non-selective topical) as an adjunct treatment if first step interventions do not give satisfactory results

- Topical non-selective NSAIDs for knee OA

8. Weakest priority recommendation (10/10)\# for strong opioids as an adjunct treatment if first step interventions or other pharmacological interventions do not give satisfactory results

- Strong opioids

! Recommendation against the prescription of glucosamine - Glucosamine

! Recommendation against the prescription of hyaluronic acid injection - Hyaluronic acid

! Recommendation against the prescription of glucocorticoid injection for hip OA

- Glucocorticoid injection for hip OA

\begin{tabular}{|c|c|c|c|c|c|}
\hline 18 & 23 & 5.0 (4.2 to 5.9$)$ & 47 & 48 & $0.6(-0.0$ to 1.1$)$ \\
\hline 9.1 & 8.9 & $-0.2(-0.8$ to 0.4$)$ & 3.3 & 3.6 & 0.3 (0.0 to 0.5$)$ \\
\hline 63 & 55 & $-8.6(-9.6$ to -7.6$)$ & 32 & 30 & $-2.7(-3.3$ to -2.2$)$ \\
\hline 11 & 11 & $0.2(-0.6$ to 0.9$)$ & 8.2 & 8.7 & 0.5 (0.1 to 0.8$)$ \\
\hline 50 & 51 & 0.6 (-0.3 to 1.7$)$ & 30 & 32 & 2.1 (1.6 to 2.7 ) \\
\hline 32 & 25 & $-6.8(-7.7$ to -5.9$)$ & 20 & 17 & $-3.2(-3.7$ to -2.8$)$ \\
\hline 6.3 & 5.3 & $-1.0(-1.5$ to -0.6$)$ & 3.5 & 3.1 & $-0.3(-0.6$ to -0.2$)$ \\
\hline 10 & 13 & 2.8 (2.1 to 3.4 ) & 9.3 & 11 & 1.9 (1.5 to 2.3$)$ \\
\hline 13 & 4.1 & $-9.5(-9.8$ to -8.8$)$ & 3.3 & 1.1 & $-2.2(-2.3$ to -2.0$)$ \\
\hline 2.1 & 0.5 & $-1.6(-1.8$ to -1.5$)$ & 0.2 & 0.1 & $-0.1(-0.2$ to -0.1$)$ \\
\hline 8.7 & 11 & 2.1 (1.0 to 3.1 ) & 7.7 & 9.3 & 1.6 (1.0 to 2.2$)$ \\
\hline
\end{tabular}

$\Delta$ Difference between After and Before

a Guideline recommendation priority ranking according to a 1-10 graded scale, where 1 is the highest degree of priority and 10 the least degree of priority.

are further reasons why first-step priority of exercise over pharmacological treatments is recommended in the Swedish guidelines (4) and even international guidelines (21). In some OA care models, the prescription of analgesics is included in the first step of treatment, in line with the argument that with some initial analgetic medication use, exercises may be performed with greater ease, with more chances of maintenance of an exercise regimen (22). However, when applying this in a recent cluster randomized trial, there was no evidence of benefit on a patient's pain and functioning compared with the usual care for OA (22).
Approximately $50-60 \%$ of prescriptions in the OA cohort are for NSAIDs and paracetamol, approximately 20-30 percentage points ( 2 times) more than the reference cohort, with some reduction in dispensed NSAIDs but not paracetamol since the release of national guidelines in 2012. However, the SPDR only includes prescribed medications while prescription-free over-the-counter NSAIDs and paracetamol are easily accessible in Sweden, making patient self-medication likely prevalent. This is supported by general population data from $5 \mathrm{EU}$ countries where in those reporting peripheral joint OA, $47 \%$ reported use of prescription medication, 
$27 \%$ reported use of over-the-counter (OTC) medications, and $9 \%$ of patients used both (23). Therefore, it is likely that the consumption of paracetamol and NSAIDs is higher in the OA cohort than our results showed, adding potential risk for harmful side effects. This suggests that public education strategies are needed in Sweden, for example through online healthcare resources to inform the public of recommended first-step interventions for OA.

The prevalence of dispensed prescriptions for glucocorticoid intra-articular injection for knee OA before use of firststep interventions was around $11 \%$ before and after publication of the national guidelines, approximately 2 percentage points higher than the reference group. It is likely that the prevalence of intra-articular injection of glucocorticoids for knee OA is higher, bearing in mind that the SPDR may not capture single-dose clinical trialing. Considering that metaanalysis of randomized controlled trials suggests only small short-term analgesic effect compared with placebo, this mild benefit may be outweighed by the potential risk of negative side effects (24). Furthermore, a recent randomized controlled trial showed better improvement in pain and disability outcomes 1 year after physical therapy compared with glucocorticoid knee injection (25). This provides further support as to why glucocorticoid intra-articular injection for knee OA intervention should only be considered as an adjunct treatment if first-step interventions do not give acceptable symptom management. Despite national guidelines recommending against the use of intra-articular injection of glucocorticoids for hip $\mathrm{OA}$, prevalence increased after guidelines were published and attained levels on a par with their use for knee OA, which was also approximately 2 percentage points higher than the reference group. It is evident that de-implementation strategies are required to further inform clinicians against this intervention for hip OA, which is also in line with a more recent review of literature (26).

In our study, dispensed prescription of weak opioids reduced from $32 \%$ to $25 \%$ after the publication of national guidelines in 2012. This was 11 percentage points higher than the reference cohort before and 7.8 percentage points higher than the reference cohort after the release of national guidelines in 2012. A concern is the rising proportion of strong opioid prescriptions in the OA cohort despite the publication of national guidelines in 2012 as well as a similar trend in the reference cohort. Similar trends were observed in a cohort from southern Sweden within the first year after knee or hip OA diagnosis (27). Somewhat higher yearly prevalence of dispensed opioid prescriptions has been observed in another cohort from southern Sweden with older mean age and most likely in a later phase of symptomatic OA (28). With considerable safety and tolerability issues (29) along with the projected further increases in prevalence and costs $(30,31)$, further efforts to improve healthcare profession compliance with national guidelines is needed to reduce inappropriate prescription of opioids for OA.
Our findings suggest that after the publication of the national guideline recommendations against the prescription of glucosamine or hyaluronic acid injection for OA, there was good compliance in Swedish routine care. A recent systematic review and meta-analysis from the OA trial bank has confirmed that glucosamine was no better than placebo for patients' pain and function in the short and long term (32). Similarly, a meta-analysis of low-risk-of-bias studies investigating the efficacy of intra-articular injections with hyaluronic acid for OA showed no analgesic effects while inclusion of high-risk-of-bias studies resulted in a small analgesic effect compared with placebo but is confounded by increased heterogeneity and indirectness of results (33). Thus, they should not be used for OA treatment.

Our findings are novel considering the lack of comparable studies examining changes before and after introduction of national OA guidelines. A major methodological strength of our study is the linkage of national databases with good coverage and completeness representative of the Swedish context and generalizable to similar healthcare systems internationally. Completeness of the BOA register in 2008-2010 initially related to a geographical reach of 12/21 national regions in Sweden; however, national representativeness of the BOA register with data collection in all 21 national regions was first attained in 2012. Comparisons with a large national reference cohort without diagnosed OA matched by sex, age, and residence increase the strength of the study design but one can speculate that some individuals in a reference population may have OA considering its prevalence and that 1 in 3 cases with knee OA symptoms do not consult a healthcare specialist (34). One must also consider possible limitations in the study design such as the diagnostic indication for the prescription, and that the patient's actual use of dispensed analgesics or use of prescription-free analgesics as well as analgesics given during hospital care are not included in the SPDR, which must be considered pragmatically in the estimation of total use of analgesics. Dispensed analgesics in terms of the magnitude of defined daily doses was not analyzed in this study. Although the knee and hip were identified by the OA cohort as the worst affected regions, one must be pragmatic regarding the potential prescription of analgesics due to other prevalent comorbidities (1). One must also recognize the difficulty in identifying the onset of the disease in the OA cohort.

\section{Conclusion}

In Sweden, dispensed prescription of analgesics commonly occurred before initiating non-pharmacological primary care interventions for OA but reduced modestly after guideline publication, which prioritizes non-pharmacological before pharmacological interventions. Additional modest improvements occurred in the stepped-care prioritization of analgesic prescription types but future strategies are required to curb an increase in prescription of strong opioids for OA and glucocorticoid intra-articular injection for hip OA. 
Conceptualization, KG and OR; methodology, AA, KG, CZ, OR, GL-S; data curation, AA, KG, CZ, OR, GL-S; formal analysis, AA and CZ' writing - original draft preparation, AA; writing - review and editing, AA, KG, $\mathrm{CZ}$, OR, GL-S. All authors have read and agreed to the published version of the manuscript.

1. GBD 2015. Disease and Injury Incidence and Prevalence Collaborators. Global, regional, and national incidence, prevalence, and years lived with disability for 310 diseases and injuries, 1990-2015: a systematic analysis for the Global Burden of Disease Study 2015. Lancet 2016; 388(10053): 1545-1602.

2. Thorstensson C, Garellick G, Rystedt H, Dahlberg L E. Better management of patients with osteoarthritis: development and nationwide implementation of an evidence-based supported osteoarthritis self-management programme. Musculoskeletal Care 2015; 13: 67-75.

3. BOA-registret, Årsrapport 2017. Available at: https://registercentrum. blob.core.windows.net/boa/r/BOA-arsrapport-2017-SkerE9Tkt7.pdf.

4. Socialstyrelsen. Nationella riktlinjer för rörelseorganens sjukdomar 2012 Osteoporos, artros, inflammatorisk ryggsjukdom och ankyloserande spondylit, psoriasisartrit och reumatoid artrit Stöd för styrning och ledning. ISBN 978-91-87169-32-8. Available at: https://www.socialstyrelsen.se/globalassets/sharepoint-dokument/artikelkatalog/nationella-riktlinjer/2012-5-1.pdf.

5. Ludvigsson J F, Almqvist C, Bonamy A K, Ljung R, Michaëlsson K, Neovius M, et al. Registers of the Swedish total population and their use in medical research. Eur J Epidemiol 2016; 31(2): 125-36.

6. Wettermark B, Hammar N, Fored C M, Leimanis A, Otterblad Olausson P, Bergman U, et al. The new Swedish Prescribed Drug Register: opportunities for pharmacoepidemiological research and experience from the first six months. Pharmacoepidemiol Drug Saf 2007; 16(7): 726-35.

7. Statistics Sweden. Longitudinal integration database for health insurance and labour market studies (LISA). Available at: https://www.scb.se/varatjanster/bestalla-mikrodata/vilka-mikrodata-finns/longitudinella-register/ longitudinell-integrationsdatabas-for-sjukforsakrings\%2D\%2Docharbetsmarknadsstudier-lisa/.

8. Altman R, Asch E, Bloch D, Bole G, Borenstein D, Brandt K, et al. Development of criteria for the classification and reporting of osteoarthritis: classification of osteoarthritis of the knee. Diagnostic and therapeutic criteria Committee of the American Rheumatism Association. Arthritis Rheum 1986; 29(8): 1039-49.

9. Altman R, Alarcon G, Appelrouth D, Bloch D, Borenstein D, Brandt $\mathbf{K}$, et al. The American College of Rheumatology criteria for the classification and reporting of osteoarthritis of the hip. Arthritis Rheum 1991; 34(5): 505-14.

10. Jensen M P, Turner J A, Romano J M, Fisher L D. Comparative reliability and validity of chronic pain intensity measures. Pain 1999; 83: 157-62.

11. Gustafsson K, Rolfson O, Eriksson M, Dahlberg L, Kvist J. Study protocol for an observational register-based study on health and risk factors in patients with hip and knee osteoarthritis. BMJ Open 2018; 8(10): e022812.

12. WHO Collaborating Centre for Drug Statistics Methodology; 2021. Available at: https://www.whocc.no/atc/structure_and_principles/.

13. Kingsbury S R, Hensor E M, Walsh C A, Hochberg M C, Conaghan P G. How do people with knee osteoarthritis use osteoarthritis pain medications and does this change over time? Data from the Osteoarthritis Initiative. Arthritis Res Ther 2013; 15(5): R106.

14. Appleyard T, Ashworth J, Bedson J, Yu D, Peat G. Trends in gabapentinoid prescribing in patients with osteoarthritis: a United Kingdom national cohort study in primary care. Osteoarthritis Cartilage 2019; 27(10): 1437-44.

15. Henriksen M, Hansen J B, Klokker L, Bliddal H, Christensen R. Comparable effects of exercise and analgesics for pain secondary to knee osteoarthritis: a meta-analysis of trials included in Cochrane systematic reviews. J Comp Eff Res 2016; 5(4): 417-31.

16. Mazzei D R, Ademola A, Abbott J H, Sajobi T, Hildebrand K, Marshall D A. Are education, exercise and diet interventions a cost-effective treatment to manage hip and knee osteoarthritis? A systematic review. Osteoarthritis Cartilage 2020: S1063-4584(20)31165-1.
17. Lear S A, Hu W, Rangarajan S, Gasevic D, Leong D, Iqbal R, et al. The effect of physical activity on mortality and cardiovascular disease in 130000 people from 17 high-income, middle-income, and low-income countries: the PURE study. Lancet 2017; 390(10113): 2643-54.

18. Rannou F, Pelletier J P, Martel-Pelletier J. Efficacy and safety of topical NSAIDs in the management of osteoarthritis: evidence from real-life setting trials and surveys. Semin Arthritis Rheum 2016; 45(4 Suppl.): S18-21.

19. Roberts E, Delgado Nunes V, Buckner S, Latchem S, Constanti M, Miller P, et al. Paracetamol: not as safe as we thought? A systematic literature review of observational studies. Ann Rheum Dis 2016; 75(3): $552-9$.

20. Conaghan P G, Arden N, Avouac B, Migliore A, Rizzoli R. Safety of paracetamol in osteoarthritis: what does the literature say? Drugs and Aging 2019; 36: 7-14.

21. Bannuru R R, Osani M C, Vaysbrot E E, Arden N K, Bennell K, Bierma-Zeinstra S M A, et al. OARSI guidelines for the non-surgical management of knee, hip, and polyarticular osteoarthritis. Osteoarthritis Cartilage 2019; 27(11): 1578-89.

22. Dziedzic K S, Healey E L, Porcheret M, Afolabi E K, Lewis M, Morden A, et al. Implementing core NICE guidelines for osteoarthritis in primary care with a model consultation (MOSAICS): a cluster randomised controlled trial. Osteoarthritis Cartilage 2018; 26(1): 43-53.

23. Kingsbury S R, Gross H J, Isherwood G, Conaghan P G. Osteoarthritis in Europe: impact on health status, work productivity and use of pharmacotherapies in five European countries. Rheumatology (Oxford) 2014; 53(5): 937-47.

24. Juni P, Hari R, Rutjes A W, Fischer R, Silletta M G, Reichenbach S, et al. Intra-articular corticosteroid for knee osteoarthritis. Cochrane Database Syst Rev 2015: CD005328.

25. Deyle G D, Allen C S, Allison S C, Gill N W, Hando B R, Petersen E J, et al. Physical therapy versus glucocorticoid injection for osteoarthritis of the knee. N Engl J Med 2020; 382(15): 1420-9.

26. McCabe P S, Maricar N, Parkes M J, Felson D T, O'Neill T W. The efficacy of intra-articular steroids in hip osteoarthritis: a systematic review. Osteoarthritis Cartilage 2016; 24: 1509-17.

27. Thorlund J B, Turkiewicz A, Prieto-Alhambra D, Englund M. Inappropriate opioid dispensing in patients with knee and hip osteoarthritis: a population-based cohort study. Osteoarthritis Cartilage 2020; 28(2): 146-53.

28. Thorlund J B, Turkiewicz A, Prieto-Alhambra D, Englund M. Opioid use in knee or hip osteoarthritis: a region-wide population-based cohort study. Osteoarthritis Cartilage 2019; 27(6): 871-7.

29. Fuggle N, Curtis E, Shaw S, Spooner L, Bruyére O, Ntani G, et al. Safety of opioids in osteoarthritis: outcomes of a systematic review and meta-analysis. Drugs Aging 2019; 36(Suppl. 1): 129-43.

30. Turkiewicz A, Petersson I F, Björk J, Hawker G, Dahlberg L E, Lohmander L S, et al. Current and future impact of osteoarthritis on health care: a population-based study with projections to year 2032 . Osteoarthritis Cartilage 2014; 22(11): 1826-32.

31. Ackerman I N, Zomer E, Gilmartin-Thomas J F, Liew D. Forecasting the future burden of opioids for osteoarthritis. Osteoarthritis Cartilage 2018; 26(3): 350-5.

32. Runhaar J, Rozendaal R M, van Middelkoop M, Bijlsma H J W, Doherty M, Dziedzic K S, et al. Subgroup analyses of the effectiveness of oral glucosamine for knee and hip osteoarthritis: a systematic review and individual patient data meta-analysis from the OA trial bank. Ann Rheum Dis 2017; 76(11): 1862-9.

33. Johansen M, Bahrt H, Altman R D, Bartels E M, Juhl C B, Bliddal $\mathbf{H}$, et al. Exploring reasons for the observed inconsistent trial reports on intra-articular injections with hyaluronic acid in the treatment of osteoarthritis: meta-regression analyses of randomized trials. Semin Arthritis Rheum 2016; 46(1): 34-48.

34. Turkiewicz A, Gerhardsson de Verdier M, Engström G, Nilsson P M, Mellström C, Lohmander L S, et al. Prevalence of knee pain and knee OA in southern Sweden and the proportion that seeks medical care. Rheumatology (Oxford) 2015; 54(5): 827-35. 


\section{Supplementary data}

Table 1. ATC codes for medications extracted from the SPDR

\begin{tabular}{|c|c|c|c|c|}
\hline NSAIDS & Paracetamol & Opioids & $\begin{array}{l}\text { Other joint pain } \\
\text { related analgesics }\end{array}$ & Antiepileptics \\
\hline $\begin{array}{c}\text { Non-selective } \\
\text { M01AB01 } \\
\text { M01AB02 } \\
\text { M01AB05 } \\
\text { M01AB55 } \\
\text { M01AC01 } \\
\text { M01AC02 } \\
\text { M01AC05 } \\
\text { M01AC06 } \\
\text { M01AE01 } \\
\text { M01AE02 } \\
\text { M01AE03 } \\
\text { M01AE14 } \\
\text { M01AE52 } \\
\text { N02BA01 } \\
\text { N02BA51 } \\
\text { N02BB01 } \\
\text { N02BB51 } \\
\text { Non-selective } \\
\text { M02AA10 } \\
\text { M02AA13 } \\
\text { M02AA15 } \\
\text { COX-2 inhibito } \\
\text { M01AH01 } \\
\text { M01AH05 } \\
\text { M01AH06 } \\
\text { M01AX01 }\end{array}$ & $\begin{array}{l}\text { N02BE01 } \\
\text { N02BE51 }\end{array}$ & $\begin{array}{c}\text { Weak opioids } \\
\text { N02AA59 } \\
\text { N02AC04 } \\
\text { N02AE01 } \\
\text { N02AJ06 } \\
\text { N02AJ08 } \\
\text { N02AJ09 } \\
\text { N02AX02 } \\
\text { N02AX06 } \\
\text { Strong opioids } \\
\text { N02AA01 } \\
\text { N02AA03 } \\
\text { N02AA05 } \\
\text { N02AA55 } \\
\text { N02AB01 } \\
\text { N02AB03 } \\
\text { N02AC04 } \\
\text { N02AE01 } \\
\text { N02AG01 } \\
\text { N02AG02 }\end{array}$ & $\begin{array}{l}\text { M01AX01 } \\
\text { M01AX05 } \\
\text { M09AX01 } \\
\text { H02AB01 } \\
\text { H02AB02 } \\
\text { H02AB04 } \\
\text { H02AB06 } \\
\text { H02AB07 } \\
\text { H02AB08 } \\
\text { H02AB09 }\end{array}$ & $\begin{array}{l}\text { N03AX12 } \\
\text { N03AX16 }\end{array}$ \\
\hline
\end{tabular}

